\title{
Optimal Control Problems Constrained by the Stochastic Navier- Stokes Equations with Multiplicative Lévy Noise
}

\author{
Peter Benner * and Christoph Trautwein ** \\ Max Planck Institute for Dynamics of Complex Technical Systems, Sandtorstraße 1, 39106 Magdeburg, Ger- \\ many
}

Received XXXX, revised XXXX, accepted XXXX

Published online XXXX

Key words Stochastic Navier-Stokes equations, Stochastic optimal control, Lévy process, Local mild solution MSC (2010) 93E20, 49J20, 35Q30

\begin{abstract}
We consider the stochastic Navier-Stokes equations in a multidimensional domain, where the noise term allows jumps and where the action of a control in order to influence the dynamics is included. In order to prove existence and uniqueness of an optimal control w.r.t. a given cost functional, we first need to show the existence and uniqueness of a local mild solution of the considered stochastic Navier-Stokes equations. We then discuss the control problem, where the cost functional includes stopping times dependent on controls. Based on the continuity of the cost functional, we can apply existence and uniqueness results provided in [1], which enables us to show that a unique optimal control exists.
\end{abstract}

Copyright line will be provided by the publisher

\section{Introduction}

In this paper, we discuss an optimal control problem for the unsteady Navier-Stokes equations influenced by noise terms. Concerning fluid dynamics, noise may enter the system due to structural vibration and other environmental effects, where we assume that jumps are allowed. The aim is to control flow fields affected by noise, where we incorporate physical requirements, such as drag minimization, lift enhancement, mixing enhancement, turbulence minimization and stabilization, see [2] and the references therein; see also [3].

Before turning our attention to the actual optimal control problem, it is first necessary to write the NavierStokes equations in a form containing a control input and a noise term. This is done by writing the external forcing in a specific form, which results then in an initial value problem for the controlled stochastic NavierStokes equations. In order to prove existence and uniqueness of an optimal control for a given cost functional, one first needs to ensure existence and uniqueness for this initial value problem for all feasible initial values and a given fixed control. As we argue below, the necessary existence and uniqueness result for the controlled stochastic Navier-Stokes equations has not been available in the literature so far. Therefore, we first turn our attention to this problem.

A large number of authors consider the initial value problem of the deterministic Navier-Stokes equations by an $L_{2}$-space approach. In [2, 4], the existence and uniqueness of global weak solutions for two-dimensional domains are proved by using a Galerkin approximation. For three-dimensional domains, uniqueness is still an open problem. Using semigroup theory, the existence of a unique local solution was proved in [5]. Under some conditions on the initial data and the external force, the result was extended to a global solution. A generalization in terms of $L_{r}$ theory can be found in [6,7].

In the last decades, existence and uniqueness results of solutions to the stochastic Navier-Stokes equations have been studied extensively. Unique weak solutions of the stochastic Navier-Stokes equations exist only for two-dimensional domains. In [8,9], weak solutions are considered with noise terms given by Wiener processes. Weak solutions with Lévy noise are considered in [10,11]. For three-dimensional domains, uniqueness is still

\footnotetext{
* E-mail: benner@mpi-magdeburg.mpg.de

** Corresponding author E-mail: trautwein@mpi-magdeburg.mpg.de
} 
an open problem and weak solutions are introduced as martingale solutions, see [12-16]. Another approach is given by using semigroup theory leading to the definition of mild solutions. The existence and uniqueness of a mild solution over an arbitrary time interval can be obtained under certain additional assumptions, see [17 [18]. In general, a unique mild solution of the stochastic Navier-Stokes equations does not exist. Thus, stopping times are required to define local mild solutions. For the local mild solution with additive noise given by Wiener processes, we refer to [19]. In [20, 21], the stochastic Navier-Stokes equations with additive Lévy noise are considered. In [22], an existence and uniqueness result for strong pathwise solutions is given. For further definitions of solutions to the fractional stochastic Navier-Stokes equations, we refer to [18].

We generalize the results in the sense that we consider a local mild solution of the multidimensional stochastic Navier-Stokes equations with multiplicative Lévy noise. Thus, we involve especially two-dimensional as well as three-dimensional domains and the noise may depend on the state. Using $L_{2}$ theory, we first analyze a modified system to obtain the existence and uniqueness of a mild solution over an arbitrary time interval by applying a Banach fixed point theorem. Based on the mild solution of this modified system, we can introduce stopping times and prove the existence and uniqueness of the local mild solution to the stochastic Navier-Stokes equations. Under certain additional assumptions, these stopping times are almost surely equal to an arbitrary terminal point and therefore, the local mild solution becomes a global mild solution.

The cost functional introduced in this paper is motivated by common control strategies. In [23-26], the problem is formulated as a tracking type problem arising in data assimilation. Approaches that minimize the enstrophy can be found in [2 27 28]. In [29], the cost functional combines both strategies by introducing weights. The shortcoming of these papers is the restriction to two-dimensional domains. Here, we remove this limitation and using the theory of fractional power operators, we formulate a control problem such that under certain conditions, the special cases mentioned can be obtained. Moreover, we have to pay particular attention to the fact that in general the solution exists only locally in time. Hence, the cost functional has to contain stopping times to be well defined. Due to the existence and uniqueness result of the solution, the state as well as the stopping times depend on the controls. This is the major difficulty when analyzing the properties of the cost functional. However, we show that the state as well as the stopping times are continuous with respect to the control. Therefore, we can conclude that the cost functional is continuous, and we prove the existence and uniqueness of optimal controls based on results given in [1].

The main contribution of this paper is an existence and uniqueness result of a local mild solution to the stochastic Navier-Stokes equations with multiplicative Lévy noise stated in Theorem 4.6 and Theorem 4.7 Here, we involve especially two-dimensional as well as three-dimensional domains. Moreover, we analyze a control problem, where the cost functional includes stopping times dependent on controls. Based on a continuity result, we get the existence and uniqueness of optimal controls stated in Theorem 5.2

The paper is organized as follows. In Section 2, we reformulate the deterministic Navier-Stokes equations in an abstract form. Moreover, we state some well known properties of the Stokes operator as well as for the nonlinear term. A brief introduction of Hilbert space valued Lévy processes and the properties of stochastic integrals are given in Section 3 for better readability. Section 4 addresses the existence and uniqueness of a local mild solution to the stochastic Navier-Stokes equations with multiplicative Lévy noise. In Section 5, we prove the continuity of the cost functional with respect to the control, which enables us to conclude that unique optimal controls exist.

\section{Preliminaries}

Throughout this paper, let $\mathcal{D} \subset \mathbb{R}^{n}, n \geq 2$, be a connected and bounded domain with $C^{\infty}$ boundary $\partial \mathcal{D}$. We consider the following Navier-Stokes equations with Dirichlet boundary condition:

$$
\left\{\begin{aligned}
\frac{\partial}{\partial t} y(t, x)+(y(t, x) \cdot \nabla) y(t, x)+\nabla p(t, x)-\nu \Delta y(t, x) & =f(t, x) & & \text { in }(0, T) \times \mathcal{D}, \\
\operatorname{div} y(t, x) & =0 & & \text { in }(0, T) \times \mathcal{D}, \\
y(t, x) & =0 & & \text { on }(0, T) \times \partial \mathcal{D}, \\
y(0, x) & =\xi(x) & & \text { in } \mathcal{D},
\end{aligned}\right.
$$


where $y(t, x) \in \mathbb{R}^{n}$ denotes the velocity field with initial value $\xi(x) \in \mathbb{R}^{n}$ and $p(t, x) \in \mathbb{R}$ describes the pressure of the fluid. The parameter $\nu>0$ is the viscosity parameter (for the sake of simplicity, we assume $\nu=1$ ) and $f(t, x) \in \mathbb{R}^{n}$ is the external force.

Next, we state the Navier-Stokes equations in an abstract form. For more details, we refer to [7]. For $s \geq 0$, we denote by $H^{s}(\mathcal{D})$ the usual Sobolev space and for $s \geq \frac{1}{2}$, we set $H_{0}^{s}(\mathcal{D})=\left\{y \in H^{s}(\mathcal{D}): y=0\right.$ on $\left.\partial \mathcal{D}\right\}$. We introduce the following common spaces:

$$
\begin{aligned}
H & =\text { Completion of }\left\{y \in\left(C_{0}^{\infty}(\mathcal{D})\right)^{n}: \operatorname{div} y=0 \text { in } \mathcal{D}\right\} \text { in }\left(L^{2}(\mathcal{D})\right)^{n} \\
& =\left\{y \in\left(L^{2}(\mathcal{D})\right)^{n}: \operatorname{div} y=0 \text { in } \mathcal{D}, y \cdot \eta=0 \text { on } \partial \mathcal{D}\right\}, \\
V & =\text { Completion of }\left\{y \in\left(C_{0}^{\infty}(\mathcal{D})\right)^{n}: \operatorname{div} y=0 \text { in } \mathcal{D}\right\} \text { in }\left(H^{1}(\mathcal{D})\right)^{n} \\
& =\left\{y \in\left(H_{0}^{1}(\mathcal{D})\right)^{n}: \operatorname{div} y=0 \text { in } \mathcal{D}\right\},
\end{aligned}
$$

where $\eta$ denotes the unit outward normal to $\partial \mathcal{D}$. The spaces $H$ equipped with the inner product

$$
\langle y, z\rangle_{H}=\langle y, z\rangle_{\left(L^{2}(\mathcal{D})\right)^{n}}=\int_{\mathcal{D}} \sum_{i=1}^{n} y_{i}(x) z_{i}(x) d x
$$

for every $y=\left(y_{1}, \ldots, y_{n}\right), z=\left(z_{1}, \ldots, z_{n}\right) \in H$ becomes a Hilbert space. For all $x=\left(x_{1}, \ldots, x_{n}\right) \in \mathcal{D}$, we denote $D^{j}=\frac{\partial^{|j|}}{\partial x_{1}^{j_{1}} \ldots \partial x_{n}^{j_{n}}}$ with $|j|=\sum_{i=1}^{n} j_{i}$. We set $D^{j} y=\left(D^{j} y_{1}, \ldots, D^{j} y_{n}\right)$ for every $y=\left(y_{1}, \ldots, y_{n}\right) \in V$ and $|j| \leq 1$. Then the space $V$ equipped with the inner product

$$
\langle y, z\rangle_{V}=\sum_{|j| \leq 1}\left\langle D^{j} y, D^{j} z\right\rangle_{\left(L^{2}(\mathcal{D})\right)^{n}}
$$

for every $y, z \in V$ becomes a Hilbert space. The norm in $H$ and $V$ is denoted by $\|\cdot\|_{H}$ and $\|\cdot\|_{V}$, respectively. Moreover, we get the orthogonal Helmholtz decomposition

$$
\left(L^{2}(\mathcal{D})\right)^{n}=H \oplus\left\{\nabla y: y \in H^{1}(\mathcal{D})\right\},
$$

where $\oplus$ denotes the direct sum. Then there exists an orthogonal projection $\Pi:\left(L^{2}(\mathcal{D})\right)^{n} \rightarrow H$, see [30]. Next, we define the Stokes Operator $A: D(A) \subset H \rightarrow H$ by $A y=-\Pi \Delta y$ for every $y \in D(A)$, where $\Delta$ denotes the Laplace operator. The domain is given by

$$
D(A)=\left(H^{2}(\mathcal{D})\right)^{n} \cap V .
$$

We have the following properties of the Stokes operator, see [7, 31-33].

Lemma 2.1 The Stokes operator $A: D(A) \subset H \rightarrow H$ is positive, self adjoint and has a bounded inverse. Moreover, the operator $-A$ is the infinitesimal generator of an analytic semigroup $\left(e^{-A t}\right)_{t \geq 0}$ such that we have $\left\|e^{-A t}\right\|_{\mathcal{L}(H)} \leq 1$ for all $t \geq 0$.

Due to the previous Lemma, we can introduce fractional powers of the Stokes operator, see [33-35]. For $\alpha>0$, we define

$$
A^{-\alpha}=\frac{1}{\Gamma(\alpha)} \int_{0}^{\infty} t^{\alpha-1} e^{-A t} d t
$$

where $\Gamma(\cdot)$ denotes the gamma function. The operator $A^{-\alpha}$ is linear, bounded and invertible in $H$. Hence, we define for all $\alpha>0$

$$
A^{\alpha}=\left(A^{-\alpha}\right)^{-1} \text {. }
$$

Moreover, we set $A^{0}=I$, where $I$ is the identity operator in $H$. For $\alpha>0$, the operator $A^{\alpha}$ is linear and closed in $H$ with dense domain $D\left(A^{\alpha}\right)=R\left(A^{-\alpha}\right)$, where $R\left(A^{-\alpha}\right)$ denotes the range of $A^{-\alpha}$. Next, we provide some useful properties of fractional powers of the Stokes operator. Let us denote the resolvent set of the operator $A$ by $\rho(A)$. Then we have the following result. 
Lemma 2.2 (cf. Section 2.6, [34]) We have

(i) for $\alpha, \beta \in \mathbb{R}$, we have $A^{\alpha+\beta} y=A^{\alpha} A^{\beta}$ y for every $y \in D\left(A^{\gamma}\right)$, where $\gamma=\max \{\alpha, \beta, \alpha+\beta\}$,

(ii) $e^{-A t}: H \rightarrow D\left(A^{\alpha}\right)$ for all $t>0$ and $\alpha \geq 0$,

(iii) we have $A^{\alpha} e^{-A t} y=e^{-A t} A^{\alpha} y$ for every $y \in D\left(A^{\alpha}\right)$ with $\alpha \in \mathbb{R}$,

(iv) the operator $A^{\alpha} e^{-A t}$ is bounded for all $t>0$ and there exist constants $M_{\alpha}, \theta>0$ such that

$$
\left\|A^{\alpha} e^{-A t}\right\|_{\mathcal{L}(H)} \leq M_{\alpha} t^{-\alpha} e^{-\theta t}
$$

(v) $0 \leq \beta \leq \alpha \leq 1$ implies $D\left(A^{\alpha}\right) \subset D\left(A^{\beta}\right)$ and there exists a constant $C>0$ such that for every $y \in D\left(A^{\alpha}\right)$

$$
\left\|A^{\beta} y\right\|_{H} \leq C\left\|A^{\alpha} y\right\|_{H} .
$$

Thus, we obtain that the space $D\left(A^{\alpha}\right)$ for $\alpha \in(0,1]$ equipped with the inner product

$$
\langle y, z\rangle_{D\left(A^{\alpha}\right)}=\left\langle A^{\alpha} y, A^{\alpha} z\right\rangle_{H}
$$

for every $y, z \in D\left(A^{\alpha}\right)$ becomes a Hilbert space.

Next, we specify the domain of the operator $A^{\alpha}$ for $\alpha \in(0,1)$ in terms of Sobolev spaces. Let the operator $A_{D}: D\left(A_{D}\right) \subset\left(L^{2}(\mathcal{D})\right)^{n} \rightarrow\left(L^{2}(\mathcal{D})\right)^{n}$ be the Laplace operator with homogeneous Dirichlet boundary condition given by $A_{D} y=-\Delta y$ for every $y \in D\left(A_{D}\right)$. The domain is given by

$$
D\left(A_{D}\right)=\left(H_{0}^{1}(\mathcal{D})\right)^{n} \cap\left(H^{2}(\mathcal{D})\right)^{n} .
$$

Then $A_{D}$ is a positive and self adjoint operator and $-A_{D}$ is the infinitesimal generator of an analytic semigroup $\left(e^{-A_{D} t}\right)_{t \geq 0}$ such that $\left\|e^{-A_{D} t}\right\|_{\mathcal{L}\left(\left(L^{2}(\mathcal{D})\right)^{n}\right)} \leq 1$ for all $t \geq 0$. Hence, we can define fractional powers and we get the following result.

Proposition 2.3 (Theorem 1.1, [31]) Let the operator $A: D(A) \subset H \rightarrow H$ be the Stokes Operator and let $A_{D}: D\left(A_{D}\right) \subset\left(L^{2}(\mathcal{D})\right)^{n} \rightarrow\left(L^{2}(\mathcal{D})\right)^{n}$ be the Laplace operator with homogeneous Dirichlet boundary condition. Then, we have for any $\alpha \in(0,1)$

$$
D\left(A^{\alpha}\right)=D\left(A_{D}^{\alpha}\right) \cap H .
$$

The domain of $A_{D}^{\alpha}$ can be determined explicitly for $\alpha \in(0,1)$.

Proposition 2.4 (cf. Theorem 1, [36]) Let $A_{D}: D\left(A_{D}\right) \subset\left(L^{2}(\mathcal{D})\right)^{n} \rightarrow\left(L^{2}(\mathcal{D})\right)^{n}$ be the Laplace operator with homogeneous Dirichlet boundary condition. Then we have

(i) $D\left(A_{D}^{\alpha}\right)=\left(H^{2 \alpha}(\mathcal{D})\right)^{n}$ for $0<\alpha<\frac{1}{4}$,

(ii) $D\left(A_{D}^{1 / 4}\right) \subset\left(H^{1 / 2}(\mathcal{D})\right)^{n}$,

(iii) $D\left(A_{D}^{\alpha}\right)=\left(H_{0}^{2 \alpha}(\mathcal{D})\right)^{n}$ for $\frac{1}{4}<\alpha<\frac{3}{4}$,

(ii) $D\left(A_{D}^{3 / 4}\right) \subset\left(H_{0}^{3 / 2}(\mathcal{D})\right)^{n}$,

(v) $D\left(A_{D}^{\alpha}\right)=\left(H_{0}^{2 \alpha}(\mathcal{D})\right)^{n}$ for $\frac{3}{4}<\alpha<1$.

We define $B(y, z)=\Pi(y \cdot \nabla) z$ for some $y, z \in H$. If $y=z$, we write $B(y)=B(y, y)$. Applying the projection $\Pi$, the above Navier-Stokes equations can be formulated in the following abstract form:

$$
\left\{\begin{aligned}
\frac{d}{d t} y(t) & =-A y(t)-B(y(t))+f(t) \quad \text { in }(0, T), \\
y(0) & =\xi
\end{aligned}\right.
$$


where we assume $\xi, f(t) \in H$. According to [7], one can consider this equation in integral form

$$
y(t)=e^{-A t} \xi-\int_{0}^{t} e^{-A(t-s)}[B(y(s))-f(s)] d s
$$

for $t \in[0, T]$. In general, existence and uniqueness results of a solution to system (1) can not be obtained over the time interval $[0, T]$. However, for a certain point of time $T_{*} \in(0, T]$, there exists a unique solution of system (1) on the time interval $\left[0, T_{*}\right]$, see [7, Theorem 2.3] and the references therein. In the remaining part of this section, we recall some useful results for dealing with the nonlinear term in equation (1).

Lemma 2.5 (Lemma 2.2, |7|) Let $0 \leq \delta<\frac{1}{2}+\frac{n}{4}$. If $y \in D\left(A^{\alpha_{1}}\right)$ and $z \in D\left(A^{\alpha_{2}}\right)$, then we have

$$
\left\|A^{-\delta} B(y, z)\right\|_{H} \leq \widetilde{M}\left\|A^{\alpha_{1}} y\right\|_{H}\left\|A^{\alpha_{2}} z\right\|_{H},
$$

with some constant $\widetilde{M}=\widetilde{M}_{\delta, \alpha_{1}, \alpha_{2}}$, provided that $\alpha_{1}, \alpha_{2}>0, \delta+\alpha_{2}>\frac{1}{2}$ and $\delta+\alpha_{1}+\alpha_{2} \geq \frac{n}{4}+\frac{1}{2}$.

Corollary 2.6 Let $\alpha_{1}, \alpha_{2}$ and $\delta$ be as in Lemma 2.5. If $y, z \in D\left(A^{\beta}\right), \beta=\max \left\{\alpha_{1}, \alpha_{2}\right\}$, then we have

$$
\left\|A^{-\delta}(B(y)-B(z))\right\|_{H} \leq \widetilde{M}\left(\left\|A^{\alpha_{1}} y\right\|_{H}\left\|A^{\alpha_{2}}(y-z)\right\|_{H}+\left\|A^{\alpha_{1}}(y-z)\right\|_{H}\left\|A^{\alpha_{2}} z\right\|_{H}\right) .
$$

\section{Lévy processes in Hilbert Spaces and the Stochastic Integral}

In this section, we give a brief introduction to stochastic integrals, where the noise term is defined as a Hilbert space valued Lévy process. For more details, see [37].

Throughout this paper, let $(\Omega, \mathcal{F}, \mathbb{P})$ be a complete probability space endowed with a filtration $\left(\mathcal{F}_{t}\right)_{t \in[0, T]}$ satisfying $\mathcal{F}_{t}=\bigcap_{s>t} \mathcal{F}_{s}$ for all $t \in[0, T]$ and $\mathcal{F}_{0}$ contains all sets of $\mathcal{F}$ with $\mathbb{P}$-measure 0 . We start with the formal definition of a Hilbert space valued Lévy process and some basic properties.

Definition 3.1 (cf. Definition 4.1, [37]) A stochastic process $(L(t))_{t \in[0, T]}$ taking values in a Hilbert space $E$ is called a Lévy process if

- $\mathbb{P}$-a.s. $L(0)=0$,

- $(L(t))_{t \in[0, T]}$ has independent and homogeneous increments, and

- $(L(t))_{t \in[0, T]}$ is stochastically continuous, i.e. for all $t \in[0, T]$ and $\varepsilon>0$

$$
\lim _{s \rightarrow t} \mathbb{P}\left(\|L(s)-L(t)\|_{E}>\varepsilon\right)=0 .
$$

Definition 3.2 (cf. Definition 3.16, [37]) A stochastic process $(L(t))_{t \in[0, T]}$ taking values in a Hilbert space $E$ is called a càdlàg process if

- $(L(t))_{t \in[0, T]}$ is right-continuous, i.e. $L(t+)=\lim _{s \downarrow t} L(s)=L(t)$ for all $t \in[0, T]$ and

- $(L(t))_{t \in[0, T]}$ has left limits, i.e. $L(t-)=\lim _{s \uparrow t} L(s)$ exists for all $t \in[0, T]$.

Proposition 3.3 (cf. Theorem 4.3, [37]) For every Lévy process $(L(t))_{t \in[0, T]}$ there exists a càdlàg process $(\tilde{L}(t))_{t \in[0, T]}$ such that $\mathbb{P}(L(t)=\tilde{L}(t))=1$ for all $t \in[0, T]$.

Proposition 3.4 (Theorem 4.47 (i), [37]) A Lévy process $(L(t))_{t \in[0, T]}$ on a Hilbert space E is square integrable if and only if its Lévy measure $\nu$ satisfies

$$
\int_{E}\|y\|_{E}^{2} \nu(d y)<\infty
$$

We denote by $\mathcal{L}_{1}(E)$ the space of all nonnegative definite symmetric nuclear operators on $E$. Then we get the following result. 
Proposition 3.5 (Theorem 4.44, [37]) Let $(L(t))_{t \in[0, T]}$ be a square integrable Lévy process. Then there exist $m \in E$ and $Q \in \mathcal{L}_{1}(E)$ such that for all $t, s \in[0, T]$ and $y, z \in E$

$$
\begin{aligned}
& \mathbb{E}\langle L(t), y\rangle_{E}=\langle m, y\rangle_{E} t, \\
& \mathbb{E}\langle L(t)-m t, y\rangle_{E}\langle L(s)-m s, z\rangle_{E}=t \wedge s\langle Q y, z\rangle_{E}, \\
& \mathbb{E}\|L(t)-m t\|_{E}^{2}=t \operatorname{Tr}(Q),
\end{aligned}
$$

where $t \wedge s=\min \{t, s\}$.

In the proposition above, the element $m \in E$ is called mean and $Q \in \mathcal{L}_{1}(E)$ is called the covariance operator.

Proposition 3.6 (Theorem 4.49 (i), [37]) If the process $(L(t))_{t \in[0, T]}$ is an integrable Lévy process with mean zero, then $(L(t))_{t \in[0, T]}$ is a martingale with respect to the filtration $\left(\mathcal{F}_{t}\right)_{t \in[0, T]}$.

Remark 3.7 For a comparison with finite dimensional Lévy processes, we refer to [38].

The construction of the stochastic integral with respect to Lévy processes is similar to the case of Q-Wiener processes. For a comparison to Q-Wiener processes, we refer to [39]. First, we give a definition of predictable processes. Let $\mathcal{P}$ denote the smallest $\sigma$-field of subsets of $[0, T] \times \Omega$. Then we have the following definition.

Definition 3.8 (cf. Definition 3.4, [37]) A stochastic process $(X(t))_{t \in[0, T]}$ taking values in the measurable space $(\mathcal{X}, \mathscr{B}(\mathcal{X}))$ is called predictable if it is a measurable mapping from $([0, T] \times \Omega, \mathcal{P})$ to $(\mathcal{X}, \mathscr{B}(\mathcal{X}))$.

Every predictable process is adapted to the filtration $\left(\mathcal{F}_{t}\right)_{t \in[0, T]}$. The converse is not true in general. However, the following lemma is useful to conclude that a stochastic process has a predictable version.

Lemma 3.9 (Proposition 3.21, [37]) Assume that the stochastic process $(X(t))_{t \in[0, T]}$ is $\left(\mathcal{F}_{t}\right)_{t \in[0, T]}$ adapted and stochastically continuous. Then the process $(X(t))_{t \in[0, T]}$ has a predictable version.

Let $(L(t))_{t \in[0, T]}$ be an $E$-valued square integrable Lévy martingale, i.e. $(L(t))_{t \in[0, T]}$ is a square integrable Lévy process and a martingale with respect to $\left(\mathcal{F}_{t}\right)_{t \in[0, T]}$. We denote the covariance operator of $(L(t))_{t \in[0, T]}$ by $Q \in \mathcal{L}_{1}(E)$. Then there exists a unique operator $Q^{1 / 2} \in \mathcal{L}_{1}(E)$ such that $Q^{1 / 2} \circ Q^{1 / 2}=Q$. We denote by $\mathcal{L}_{(H S)}\left(Q^{1 / 2}(E) ; \mathcal{H}\right)$ the space of Hilbert-Schmidt operators mapping from $Q^{1 / 2}(E)$ into another Hilbert space $\mathcal{H}$. In what follows, let $(\Phi(t))_{t \in[0, T]}$ be a predictable process with values in $\mathcal{L}_{(H S)}\left(Q^{1 / 2}(E) ; \mathcal{H}\right)$ such that

$$
\mathbb{E} \int_{0}^{T}\left\|\Phi(t) Q^{1 / 2}\right\|_{\mathcal{L}_{(H S)}(E ; \mathcal{H})}^{2} d t<\infty .
$$

Then, one can define the stochastic integral

$$
\psi(t)=\int_{0}^{t} \Phi(s) d L(s)
$$

for all $t \in[0, T]$ and $\mathbb{P}$-a.s. and we have

$$
\mathbb{E}\|\psi(t)\|_{\mathcal{H}}^{2}=\mathbb{E} \int_{0}^{t}\left\|\Phi(s) Q^{1 / 2}\right\|_{\mathcal{L}_{(H S)}(E ; \mathcal{H})}^{2} d s .
$$

The following proposition is useful when dealing with a closed operator $\mathcal{A}: D(\mathcal{A}) \subset \mathcal{H} \rightarrow \mathcal{H}$.

Proposition 3.10 If $\Phi(t) y \in D(\mathcal{A})$ for every $y \in E$, all $t \in[0, T]$ and $\mathbb{P}$-almost surely,

$$
\mathbb{E} \int_{0}^{T}\left\|\Phi(t) Q^{1 / 2}\right\|_{\mathcal{L}_{(H S)}(E ; \mathcal{H})}^{2} d t<\infty \quad \text { and } \quad \mathbb{E} \int_{0}^{T}\left\|\mathcal{A} \Phi(t) Q^{1 / 2}\right\|_{\mathcal{L}_{(H S)}(E ; \mathcal{H})}^{2} d t<\infty
$$

then we have $\mathbb{P}$-a.s. $\int_{0}^{T} \Phi(t) d L(t) \in D(\mathcal{A})$ and

$$
\mathcal{A} \int_{0}^{T} \Phi(t) d L(t)=\int_{0}^{T} \mathcal{A} \Phi(t) d L(t) .
$$


Pro of. One obtains the result similarly to the case of stochastic integrals with respect to Q-Wiener processes, see [39, Proposition 4.30].

The remaining part of this section is devoted to stochastic convolutions. Let $(S(t))_{t \geq 0}$ be a $C_{0}$-semigroup in $\mathcal{H}$. Then the stochastic convolution $(\mathcal{I}(t))_{t \in[0, T]}$ given by

$$
\mathcal{I}(t)=\int_{0}^{t} S(t-s) \Phi(s) d L(s)
$$

is well defined for all $t \in[0, T]$ and $\mathbb{P}$-almost surely. Under additional assumptions, we get the following maximal inequality.

Proposition 3.11 (cf. Proposition 1.3 (i), [40]) Let the $C_{0}$-semigroup $(S(t))_{t \geq 0}$ satisfy $\|S(t)\|_{\mathcal{L}(\mathcal{H})} \leq 1$ for all $t \geq 0$. If $p \in(0,2]$, then

$$
\mathbb{E} \sup _{t \in[0, T]}\left\|\int_{0}^{t} S(t-s) \Phi(s) d L(s)\right\|_{\mathcal{H}}^{p} \leq \widetilde{C}_{p} \mathbb{E}\left(\int_{0}^{T}\left\|\Phi(t) Q^{1 / 2}\right\|_{\mathcal{L}_{(H S)}(E ; \mathcal{H})}^{2} d t\right)^{p / 2},
$$

where $\widetilde{C}_{p}>0$ is a constant.

In order to define local mild solutions to SPDEs, we need to introduce a stopped stochastic convolution. Here, we can argue as in [41, Appendix]. Let $\tau$ be a stopping time with values in $[0, T]$. We consider the stopped process $(\mathcal{I}(t \wedge \tau))_{t \in[0, T]}$, where $t \wedge \tau=\min \{t, \tau\}$. Unfortunately, the formula

$$
\mathcal{I}(t \wedge \tau)=\int_{0}^{t \wedge \tau} S(t \wedge \tau-s) \Phi(s) d L(s)
$$

is not well defined due to the fact that we integrate a process, which is not even $\left(\mathcal{F}_{t}\right)_{t \in[0, T]}$ adapted. To overcome this problem, we introduce a process $\left(\mathcal{I}_{\tau}(t)\right)_{t \in[0, T]}$ given by

$$
\mathcal{I}_{\tau}(t)=\int_{0}^{t} \mathbb{1}_{[0, \tau)}(s) S(t-s) \Phi(s \wedge \tau) d L(s)
$$

for all $t \in[0, T]$ and $\mathbb{P}$-almost surely. We get the following result.

Lemma 3.12 Let $(S(t))_{t>0}$ be a $C_{0}$-semigroup in $\mathcal{H}$ and let $\tau$ be a stopping time with values in $[0, T]$. Moreover, let the processes $(\overline{\mathcal{I}}(t))_{t \in[0, T]}$ and $\left(\mathcal{I}_{\tau}(t)\right)_{t \in[0, T]}$ be given by (4) and (5), respectively. Then, we have for all $t \in[0, T]$ and $\mathbb{P}$-almost surely

$$
S(t-t \wedge \tau) \mathcal{I}(t \wedge \tau)=\mathcal{I}_{\tau}(t)
$$

and in particular

$$
\mathcal{I}(t \wedge \tau)=\mathcal{I}_{\tau}(t \wedge \tau) .
$$

Pro of. The processes $(\mathcal{I}(t))_{t \in[0, T]}$ and $\left(\mathcal{I}_{\tau}(t)\right)_{t \in[0, T]}$ have càdlàg modifications by [37, Theorem 9.24]. The remaining part of the proof can be obtained similarly to [41, Lemma A.1].

\section{The Stochastic Navier-Stokes Equations}

Here, we assume that the external force $f(t)$ in equation $(1)$ can be decomposed as the sum of a control term and a noise term. In addition, we suppose that the noise allows jumps and is dependent on the state. This leads us to the following SPDE on $D\left(A^{\alpha}\right)$ :

$$
\left\{\begin{aligned}
d y(t) & =-[A y(t)+B(y(t))-F u(t)] d t+G(y(t)) d L(t) \\
y(0) & =\xi
\end{aligned}\right.
$$


where the initial value $\xi \in L^{2}\left(\Omega ; D\left(A^{\alpha}\right)\right)$ is assumed to be $\mathcal{F}_{0}$-measurable and the Lévy process $(L(t))_{t \in[0, T]}$ is a square integrable martingale with values in $H$ and covariance operator $Q \in \mathcal{L}_{1}(H)$. Moreover, let the set of admissible controls $U$ contain all $\left(\mathcal{F}_{t}\right)_{t \in[0, T]}$ adapted processes $(u(t))_{t \in[0, T]}$ with values in $D\left(A^{\beta}\right), \beta \in[0, \alpha]$, such that

$$
\mathbb{E} \int_{0}^{T}\|u(t)\|_{D\left(A^{\beta}\right)}^{2} d t \leq \mathcal{C}
$$

where $\mathcal{C}>0$ is a constant. Let $A: D(A) \subset H \rightarrow H$ be the Stokes operator given by $A y=-\Pi \Delta y$ for every $y \in D(A)$ and let the nonlinear operator be defined by $B(y)=\Pi(y \cdot \nabla) y$ for every $y \in D\left(A^{\alpha}\right)$. Furthermore, we assume $F \in \mathcal{L}\left(D\left(A^{\beta}\right)\right)$ and $G: H \rightarrow \mathcal{L}_{(H S)}\left(Q^{1 / 2}(H) ; D\left(A^{\alpha}\right)\right)$ is a map such that for every $y, z \in H$

$$
\begin{aligned}
& \left\|G(y) Q^{1 / 2}\right\|_{\mathcal{L}_{(H S)}\left(H ; D\left(A^{\alpha}\right)\right)} \leq \widehat{C}\left(1+\|y\|_{H}\right), \\
& \left\|(G(y)-G(z)) Q^{1 / 2}\right\|_{\mathcal{L}_{(H S)}\left(H ; D\left(A^{\alpha}\right)\right)} \leq \widehat{C}\|y-z\|_{H},
\end{aligned}
$$

where $\widehat{C}>0$ is a constant.

Remark 4.1 According to Corollary 2.3, we have $D\left(A^{\alpha}\right) \subset H$ for any $\alpha \in(0,1)$. Therefore, we seek for a mild solution of system 6 in a dense subset of the space $H$.

In general, we can not ensure the existence and uniqueness of a mild solution over an arbitrary time interval $[0, T]$ since the nonlinear term $B(\cdot)$ is only locally Lipschitz continuous. Thus, we need the following definition of a local mild solution.

Definition 4.2 (cf. Definition 3.2, [18]) Let $\tau$ be a stopping time taking values in $(0, T]$ and $\left(\tau_{m}\right)_{m \in \mathbb{N}_{0}}$ be an increasing sequence of stopping times taking values in $(0, T]$ satisfying

$$
\lim _{m \rightarrow \infty} \tau_{m}=\tau \text {. }
$$

A predictable process $(y(t))_{t \in[0, \tau)}$ with values in $D\left(A^{\alpha}\right)$ is called a local mild solution of system 6 if for fixed $m \in \mathbb{N}_{0}$

$$
\mathbb{E} \sup _{t \in\left[0, \tau_{m}\right)}\|y(t)\|_{D\left(A^{\alpha}\right)}^{2}<\infty
$$

and we have for each $m \in \mathbb{N}_{0}$, all $t \in[0, T]$ and $\mathbb{P}$-a.s.

$$
\begin{aligned}
y\left(t \wedge \tau_{m}\right)= & e^{-A\left(t \wedge \tau_{m}\right)} \xi-\int_{0}^{t \wedge \tau_{m}} A^{\delta} e^{-A\left(t \wedge \tau_{m}-s\right)} A^{-\delta} B(y(s)) d s+\int_{0}^{t \wedge \tau_{m}} e^{-A\left(t \wedge \tau_{m}-s\right)} F u(s) d s \\
& +\mathcal{I}_{\tau_{m}}(G(y))\left(t \wedge \tau_{m}\right)
\end{aligned}
$$

where

$$
\mathcal{I}_{\tau_{m}}(G(y))(t)=\int_{0}^{t} \mathbb{1}_{\left[0, \tau_{m}\right)}(s) e^{-A(t-s)} G\left(y\left(s \wedge \tau_{m}\right)\right) d L(s) .
$$

Remark 4.3 In equation (9), note that the stopped stochastic convolution is defined according to Section 3 . This requires that the integrand is a predictable process, which can be ensured only if the process $(y(t))_{t \in[0, \tau)}$ is predictable. Furthermore, the process $(y(t))_{t \in[0, \tau)}$ can also be defined equivalently as an $\left(\mathcal{F}_{t}\right)_{t \in[0, T]}$ adapted càdlàg process due to [37, Proposition 9.10].

Next, we prove the existence and uniqueness of the local mild solution to system (6), whereby we closely follow [18, Appendix A]. Therefor, we need the following result. 
Lemma 4.4 (cf. [18 42]) Let $\mathcal{B}$ be a Banach space and $m \in \mathbb{N}$ be fixed. We define $\pi_{m}: \mathcal{B} \rightarrow \mathcal{B}$ by

$$
\pi_{m}(y)= \begin{cases}y & \|y\|_{\mathcal{B}} \leq m \\ m\|y\|_{\mathcal{B}}^{-1} y & \|y\|_{\mathcal{B}}>m\end{cases}
$$

for every $y \in \mathcal{B}$. Then we have the following estimates for every $y, z \in \mathcal{B}$ :

$$
\left\|\pi_{m}(y)-\pi_{m}(z)\right\|_{\mathcal{B}} \leq 2\|y-z\|_{\mathcal{B}} \quad \text { and } \quad\left\|\pi_{m}(y)\right\|_{\mathcal{B}} \leq \min \left\{m,\|y\|_{\mathcal{B}}\right\} .
$$

Instead of system $\left[6\right.$, we first consider the following system for $t \in(0, T]$ and fixed $m \in \mathbb{N}_{0}$ :

$$
\left\{\begin{aligned}
d y_{m}(t) & =-\left[A y_{m}(t)+B\left(\pi_{m}\left(y_{m}(t)\right)\right)-F u(t)\right] d t+G\left(y_{m}(t)\right) d L(t), \\
y_{m}(0) & =\xi
\end{aligned}\right.
$$

where the map $\pi_{m}(\cdot)$ is given by $[10)$.

Definition 4.5 A predictable process $\left(y_{m}(t)\right)_{t \in[0, T]}$ with values in $D\left(A^{\alpha}\right)$ is called a mild solution of system (11) if

$$
\mathbb{E} \sup _{t \in[0, T]}\left\|y_{m}(t)\right\|_{D\left(A^{\alpha}\right)}^{2}<\infty
$$

and we have for all $t \in[0, T]$ and $\mathbb{P}$-a.s.

$$
\begin{aligned}
y_{m}(t)= & e^{-A t} \xi-\int_{0}^{t} A^{\delta} e^{-A(t-s)} A^{-\delta} B\left(\pi_{m}\left(y_{m}(s)\right)\right) d s+\int_{0}^{t} e^{-A(t-s)} F u(s) d s \\
& +\int_{0}^{t} e^{-A(t-s)} G\left(y_{m}(s)\right) d L(s) .
\end{aligned}
$$

In [37], an existence and uniqueness result is shown for mild solutions of SPDEs satisfying a weaker condition. This result can not be applied here since especially a maximal inequality for the stochastic convolution is required, which is provided by Proposition 3.11. Furthermore, note that the operator $B\left(\pi_{m}(\cdot)\right)$ is bounded and globally Lipschitz on the domain of fractional powers to the Stokes operator. Hence, an existence and uniqueness result of a mild solution to system (11) follows by applying the Banach fixed point theorem. However, we will prove this result here to justify that the solution is a predictable process. Moreover, we get constraints of the parameters, which are necessary to obtain a well defined control problem in the next section.

Theorem 4.6 Let the parameters $\alpha \in(0,1)$ and $\delta \in[0,1)$ satisfy $1>\delta+\alpha>\frac{1}{2}$ and $\delta+2 \alpha \geq \frac{n}{4}+\frac{1}{2}$. Furthermore, let $u \in U$ be fixed for $\beta \in[0, \alpha]$ such that $\alpha-\beta<\frac{1}{2}$. Then for any $\xi \in L^{2}\left(\Omega ; D\left(A^{\alpha}\right)\right)$, there exists a unique mild solution $\left(y_{m}(t)\right)_{t \in[0, T]}$ of system $\left.\sqrt{11}\right)$ for fixed $m \in \mathbb{N}_{0}$. Moreover, the process $\left(y_{m}(t)\right)_{t \in[0, T]}$ is mean square continuous in $D\left(A^{\alpha}\right)$.

Proof. Let $m \in \mathbb{N}_{0}$ be fixed and let the space $\mathcal{Z}_{T}$ contain all predictable processes $(z(t))_{t \in[0, T]}$ with values in $D\left(A^{\alpha}\right)$ such that $\mathbb{E} \sup _{t \in[0, T]}\|z(t)\|_{D\left(A^{\alpha}\right)}^{2}<\infty$. The space $\mathcal{Z}_{T}$ equipped with the norm

$$
\|z\|_{\mathcal{Z}_{T}}=\mathbb{E} \sup _{t \in[0, T]}\|z(t)\|_{D\left(A^{\alpha}\right)}^{2}
$$

for every $z \in \mathcal{Z}_{T}$ becomes a Banach space. We define for every $z \in \mathcal{Z}_{T}$, all $t \in[0, T]$ and $\mathbb{P}$-a.s.

$$
\begin{aligned}
\Phi_{m}(z)(t)= & e^{-A t} \xi-\int_{0}^{t} A^{\delta} e^{-A(t-s)} A^{-\delta} B\left(\pi_{m}(z(s))\right) d s+\int_{0}^{t} e^{-A(t-s)} F u(s) d s \\
& +\int_{0}^{t} e^{-A(t-s)} G(z(s)) d L(s) .
\end{aligned}
$$


First, we prove that $\Phi_{m}$ maps $\mathcal{Z}_{T}$ into itself. We define for every $z \in \mathcal{Z}_{T}$, all $t \in[0, T]$ and $\mathbb{P}$-a.s.

$$
\begin{array}{ll}
\psi_{1}(t)=e^{-A t} \xi, \quad \psi_{2}(z)(t)=\int_{0}^{t} A^{\delta} e^{-A(t-s)} A^{-\delta} B\left(\pi_{m}(z(s))\right) d s, \\
\psi_{3}(t)=\int_{0}^{t} e^{-A(t-s)} F u(s) d s, \quad \psi_{4}(z)(t)=\int_{0}^{t} e^{-A(t-s)} G(z(s)) d L(s) .
\end{array}
$$

Recall that $\left\|e^{-A t}\right\|_{\mathcal{L}(H)} \leq 1$ for all $t \geq[0, T]$. Due to Lemma 2.2. the process $\left(\psi_{1}(t)\right)_{t \in[0, T]}$ takes values in $D\left(A^{\alpha}\right)$ and we have

$$
\mathbb{E} \sup _{t \in[0, T]}\left\|\psi_{1}(t)\right\|_{D\left(A^{\alpha}\right)}^{2}=\mathbb{E}\left\|e^{-A t} A^{\alpha} \xi\right\|_{H}^{2} \leq \mathbb{E}\|\xi\|_{D\left(A^{\alpha}\right)}^{2} .
$$

By Lemma 2.2. Lemma 2.5 and Lemma 4.4 the process $\left(\psi_{2}(z)(t)\right)_{t \in[0, T]}$ takes values in $D\left(A^{\alpha}\right)$ and there exists a constant $c_{1}>0$ such that

$$
\begin{aligned}
\mathbb{E} \sup _{t \in[0, T]}\left\|\psi_{2}(z)(t)\right\|_{D\left(A^{\alpha}\right)}^{2} & \leq \mathbb{E} \sup _{t \in[0, T]}\left(\int_{0}^{t}\left\|A^{\alpha+\delta} e^{-A(t-s)} A^{-\delta} B\left(\pi_{m}(z(s))\right)\right\|_{H} d s\right)^{2} \\
& \leq c_{1} \mathbb{E} \sup _{t \in[0, T]}\left\|\pi_{m}(z(t))\right\|_{D\left(A^{\alpha}\right)}^{4} \\
& \leq c_{1} m^{2} \mathbb{E} \sup _{t \in[0, T]}\|z(t)\|_{D\left(A^{\alpha}\right)}^{2} .
\end{aligned}
$$

Recall that the operator $F: D\left(A^{\beta}\right) \rightarrow D\left(A^{\beta}\right)$ is linear and bounded. Using Lemma 2.2 and the Cauchy-Schwarz inequality, the process $\left(\psi_{3}(t)\right)_{t \in[0, T]}$ takes values in $D\left(A^{\alpha}\right)$ and there exists a constant $c_{2}>0$ such that

$$
\begin{aligned}
\mathbb{E} \sup _{t \in[0, T]}\left\|\psi_{3}(t)\right\|_{D\left(A^{\alpha}\right)}^{2} & \leq \mathbb{E} \sup _{t \in[0, T]}\left(\int_{0}^{t}\left\|A^{\alpha-\beta} e^{-A(t-s)} A^{\beta} F u(s)\right\|_{H} d s\right)^{2} \\
& \leq c_{2} \mathbb{E} \int_{0}^{T}\|u(t)\|_{D\left(A^{\beta}\right)}^{2} d t .
\end{aligned}
$$

Due to Lemma 2.2 and inequality (7), one can verify the assumptions of Proposition 3.10 with $\mathcal{A}=A^{\alpha}$. Hence, the process $\left(\psi_{4}(z)(t)\right)_{t \in[0, T]}$ takes values in $D\left(A^{\alpha}\right)$. Using additionally Proposition 3.11 , there exists a constant $c_{3}>0$ such that

$$
\begin{aligned}
\mathbb{E} \sup _{t \in[0, T]}\left\|\psi_{4}(z)(t)\right\|_{D\left(A^{\alpha}\right)}^{2} & =\mathbb{E} \sup _{t \in[0, T]}\left\|\int_{0}^{t} e^{-A(t-s)} A^{\alpha} G(z(s)) d L(s)\right\|_{H}^{2} \\
& \leq c_{3}\left(1+\mathbb{E} \sup _{t \in[0, T]}\|z(t)\|_{D\left(A^{\alpha}\right)}^{2}\right) .
\end{aligned}
$$

Hence, we can conclude that for fixed $z \in \mathcal{Z}_{T}$, the processes $\left(\Phi_{m}(z)(t)\right)_{t \in[0, T]}$ takes values in $D\left(A^{\alpha}\right)$ such that $\mathbb{E} \sup _{t \in[0, T]}\left\|\Phi_{m}(z)(t)\right\|_{D\left(A^{\alpha}\right)}^{2}<\infty$. To obtain that there exists a predictable version of $\left(\Phi_{m}(z)(t)\right)_{t \in[0, T]}$ for fixed $z \in \mathcal{Z}_{T}$, we need the continuity in probability. Here, we show that the process $\left(\Phi_{m}(z)(t)\right)_{t \in[0, T]}$ is continuous in mean square for fixed $z \in \mathcal{Z}_{T}$, which is a stronger formulation of continuity. In what follows, we assume w.l.o.g. $0 \leq t_{0} \leq t \leq T$. By Lemma2.2, we have

$$
\mathbb{E}\left\|\psi_{1}(t)-\psi_{1}\left(t_{0}\right)\right\|_{D\left(A^{\alpha}\right)}^{2}=\mathbb{E}\left\|\left(e^{-A\left(t-t_{0}\right)}-I\right) e^{-A t_{0}} A^{\alpha} \xi\right\|_{H}^{2} .
$$


From Lemma 2.2. Lemma 2.5 and Lemma 4.4, there exists a constant $\tilde{c}_{1}>0$ such that

$$
\begin{aligned}
& \mathbb{E}\left\|\psi_{2}(z)(t)-\psi_{2}(z)\left(t_{0}\right)\right\|_{D\left(A^{\alpha}\right)}^{2} \\
& \leq 2 \mathbb{E}\left\|\int_{0}^{t_{0}}\left(e^{-A\left(t-t_{0}\right)}-I\right) A^{\alpha+\delta} e^{-A\left(t_{0}-s\right)} A^{-\delta} B\left(\pi_{m}(z(s))\right) d s\right\|_{H}^{2} \\
& \quad+2 \mathbb{E}\left(\int_{t_{0}}^{t}\left\|A^{\alpha+\delta} e^{-A(t-s)} A^{-\delta} B\left(\pi_{m}(z(s))\right)\right\|_{H} d s\right)^{2} \\
& \leq 2 \mathbb{E}\left\|\left(e^{-A\left(t-t_{0}\right)}-I\right) \int_{0}^{t_{0}} A^{\alpha+\delta} e^{-A\left(t_{0}-s\right)} A^{-\delta} B\left(\pi_{m}(z(s))\right) d s\right\|_{H}^{2} \\
& \quad+\tilde{c}_{1}\left(t-t_{0}\right)^{2-2 \alpha-2 \delta} \sup _{t \in[0, T]}\|z(t)\|_{D\left(A^{\alpha}\right)}^{2} \cdot
\end{aligned}
$$

Due to Lemma 2.2 and the Cauchy-Schwarz inequality, there exists a constant $\tilde{c}_{2}>0$ such that

$$
\begin{aligned}
& \mathbb{E}\left\|\psi_{3}(t)-\psi_{3}\left(t_{0}\right)\right\|_{D\left(A^{\alpha}\right)}^{2} \\
& \leq 2 \mathbb{E}\left\|\int_{0}^{t_{0}}\left(e^{-A\left(t-t_{0}\right)}-I\right) A^{\alpha-\beta} e^{-A\left(t_{0}-s\right)} A^{\beta} F u(s) d s\right\|_{H}^{2} \\
& +2 \mathbb{E}\left(\int_{t_{0}}^{t}\left\|A^{\alpha-\beta} e^{-A(t-s)} A^{\beta} F u(s)\right\|_{H} d s\right)^{2} \\
& \leq 2 \mathbb{E}\left\|\left(e^{-A\left(t-t_{0}\right)}-I\right) \int_{0}^{t_{0}} A^{\alpha-\beta} e^{-A\left(t_{0}-s\right)} A^{\beta} F u(s) d s\right\|_{H}^{2} \\
& \quad+\tilde{c}_{2}\left(t-t_{0}\right)^{1+2 \beta-2 \alpha} \int_{\mathbb{E}}^{T}\|u(t)\|_{D\left(A^{\beta}\right)}^{2} d t .
\end{aligned}
$$

Let $\left(e_{i}\right)_{i \in \mathbb{N}}$ be an orthonormal basis in $H$. Using Lemma 2.2, equation 3, inequality (7) and Lebesgue's monotone convergence theorem, there exists a constant $\tilde{c}_{3}>0$ such that

$$
\begin{aligned}
& \mathbb{E}\left\|\psi_{4}(z)(t)-\psi_{4}(z)\left(t_{0}\right)\right\|_{D\left(A^{\alpha}\right)}^{2} \\
& \leq 2 \mathbb{E}\left\|\int_{0}^{t_{0}}\left(e^{-A\left(t-t_{0}\right)}-I\right) e^{-A\left(t_{0}-s\right)} A^{\alpha} G(z(s)) d L(s)\right\|_{H}^{2} \\
& \quad+2 \mathbb{E}\left\|\int_{t_{0}}^{t} e^{-A(t-s)} A^{\alpha} G(z(s)) d L(s)\right\|_{H}^{2} \\
& \leq 2 \sum_{i=1}^{\infty} \mathbb{E} \int_{0}^{t_{0}}\left\|\left(e^{-A\left(t-t_{0}\right)}-I\right) e^{-A\left(t_{0}-s\right)} A^{\alpha} G(z(s)) Q^{1 / 2} e_{i}\right\|_{H}^{2} d s \\
& \quad+\tilde{c}_{3}\left(t-t_{0}\right)\left(1+\mathbb{E} \sup _{t \in[0, T]}\|z(t)\|_{D\left(A^{\alpha}\right)}^{2}\right) .
\end{aligned}
$$


Since $\lim _{t \rightarrow t_{0}}\left\|e^{-A\left(t-t_{0}\right)} h-h\right\|_{H}=0$ holds for every $h \in H$ and using Lebesgue's dominated convergence theorem, we can infer that the processes $\left(\psi_{1}(t)\right)_{t \in[0, T]},\left(\psi_{2}(z)(t)\right)_{t \in[0, T]},\left(\psi_{3}(t)\right)_{t \in[0, T]}$ and $\left(\psi_{4}(z)(t)\right)_{t \in[0, T]}$ are mean square continuous for fixed $z \in \mathcal{Z}_{T}$. Hence, the process $\left(\Phi_{m}(z)(t)\right)_{t \in[0, T]}$ is continuous in mean square for fixed $z \in \mathcal{Z}_{T}$. Therefore, we get the continuity in probability and by Lemma 3.9, there exists a predictable version. Thus, we can conclude that $\Phi_{m}$ maps $\mathcal{Z}_{T}$ into itself.

Next, we show that $\Phi_{m}$ is a contraction on $\mathcal{Z}_{T}$. Let $T_{1, m} \in(0, T]$. Using Lemma 2.2, Corollary 2.6 and Lemma 4.4 there exists a constant $C_{1}>0$ such that for every $z_{1}, z_{2} \in \mathcal{Z}_{T}$

$$
\begin{aligned}
& \mathbb{E} \sup _{t \in\left[0, T_{1, m}\right]}\left\|\psi_{2}\left(z_{1}\right)(t)-\psi_{2}\left(z_{2}\right)(t)\right\|_{D\left(A^{\alpha}\right)}^{2} \\
& \leq \mathbb{E} \sup _{t \in\left[0, T_{1, m}\right]}\left(\int_{0}^{t}\left\|A^{\alpha+\delta} e^{-A(t-s)} A^{-\delta}\left[B\left(\pi_{m}\left(z_{1}(s)\right)\right)-B\left(\pi_{m}\left(z_{2}(s)\right)\right)\right]\right\|_{H} d s\right)^{2} \\
& \leq C_{1} m^{2} T_{1, m}^{2-2 \alpha-2 \delta} \mathbb{E} \sup _{t \in\left[0, T_{1, m}\right]}\left\|z_{1}(t)-z_{2}(t)\right\|_{D\left(A^{\alpha}\right)}^{2} .
\end{aligned}
$$

By Proposition 3.11 and inequality $\left[8\right.$, there exists a constant $C_{2}>0$ such that for every $z_{1}, z_{2} \in \mathcal{Z}_{T}$

$$
\begin{aligned}
& \mathbb{E} \sup _{t \in\left[0, T_{1, m}\right]}\left\|\psi_{4}\left(z_{1}\right)(t)-\psi_{4}\left(z_{2}\right)(t)\right\|_{D\left(A^{\alpha}\right)}^{2} \\
& =\mathbb{E} \sup _{t \in\left[0, T_{1, m}\right]}\left\|\int_{0}^{t} e^{-A(t-s)} A^{\alpha}\left[G\left(z_{1}(s)\right)-G\left(z_{2}(s)\right)\right] d L(s)\right\|_{H}^{2} \\
& \leq C_{2} T_{1, m} \mathbb{E} \sup _{t \in\left[0, T_{1, m}\right]}\left\|z_{1}(t)-z_{2}(t)\right\|_{D\left(A^{\alpha}\right)}^{2} .
\end{aligned}
$$

Consequently, we obtain for every $z_{1}, z_{2} \in \mathcal{Z}_{T}$

$$
\mathbb{E} \sup _{t \in\left[0, T_{1, m}\right]}\left\|\Phi_{m}\left(z_{1}\right)(t)-\Phi_{m}\left(z_{2}\right)(t)\right\|_{D\left(A^{\alpha}\right)}^{2} \leq K_{1, m} \mathbb{E} \sup _{t \in\left[0, T_{1, m}\right]}\left\|z_{1}(t)-z_{2}(t)\right\|_{D\left(A^{\alpha}\right)}^{2},
$$

where $K_{1, m}=2 C_{1} m^{2} T_{1, m}^{2-2 \alpha-2 \delta}+2 C_{2} T_{1, m}$. We chose $T_{1, m} \in(0, T]$ such that $K_{1, m}<1$. Applying the Banach fixed point theorem, we get a unique element $y_{m} \in \mathcal{Z}_{T}$ such that for all $t \in\left[0, T_{1, m}\right]$ and $\mathbb{P}$-a.s. $y_{m}(t)=\Phi_{m}\left(y_{m}\right)(t)$. Next, we consider for every $z \in \mathcal{Z}_{T}$, all $t \in\left[T_{1, m}, T\right]$ and $\mathbb{P}$-a.s.

$$
\begin{aligned}
\Phi_{m}(z)(t)= & e^{-A\left(t-T_{1, m}\right)} y_{m}\left(T_{1, m}\right)-\int_{T_{1, m}}^{t} A^{\delta} e^{-A(t-s)} A^{-\delta} B\left(\pi_{m}(z(s))\right) d s \\
& +\int_{T_{1, m}}^{t} e^{-A(t-s)} F u(s) d s+\int_{T_{1, m}}^{t} e^{-A(t-s)} G(z(s)) d L(s) .
\end{aligned}
$$

Again, we find $T_{2, m} \in\left[T_{1, m}, T\right]$ such that there exists a unique fixed point of the map $\Phi_{m}$ on the time interval $\left[T_{1, m}, T_{2, m}\right]$. By continuing this method, we get the existence and uniqueness of a mild solution $\left(y_{m}(t)\right)_{t \in[0, T]}$ to system $\left[11\right.$ satisfying for all $t \in[0, T]$ and $\mathbb{P}$-a.s. $y_{m}(t)=\Phi_{m}\left(y_{m}\right)(t)$.

Theorem 4.7 Let the parameters $\alpha \in(0,1), \beta \in[0, \alpha]$ and $\delta \in[0,1)$ be as in Theorem 4.6 and let $u \in U$ be fixed. Then for any $\xi \in L^{2}\left(\Omega ; D\left(A^{\alpha}\right)\right)$, there exists a unique local mild solution $(y(t))_{t \in[0, \tau)}$ of system (6). Moreover, the process $(y(t))_{t \in[0, \tau)}$ is mean square continuous in $D\left(A^{\alpha}\right)$.

Proof. By Theorem 4.6 there exists a unique mild solution $\left(y_{m}(t)\right)_{t \in[0, T]}$ to system $[11$, which is mean square continuous. Moreover, the process $\left(y_{m}(t)\right)_{t \in[0, T]}$ has a càdlàg modification, see [37, Theorem 9.24]. We still denote this modification by $\left(y_{m}(t)\right)_{t \in[0, T]}$. Next, we define a sequence of stopping times $\left(\tau_{m}\right)_{m \in \mathbb{N}_{0}}$ by

$$
\tau_{m}=\inf \left\{t \in(0, T):\left\|y_{m}(t)\right\|_{D\left(A^{\alpha}\right)}>m\right\} \wedge T,
$$


where we declare $\inf \emptyset=+\infty$. Due to the definition of the map $\pi_{m}$ given by $(10)$ and equation $(12)$, we obtain for fixed $m \in \mathbb{N}_{0}$, all $t \in[0, T]$ and $\mathbb{P}$-a.s.

$$
\begin{aligned}
y_{m}\left(t \wedge \tau_{m}\right)= & e^{-A\left(t \wedge \tau_{m}\right)} \xi-\int_{0}^{t \wedge \tau_{m}} A^{\delta} e^{-A\left(t \wedge \tau_{m}-s\right)} A^{-\delta} B\left(y_{m}(s)\right) d s+\int_{0}^{t \wedge \tau_{m}} e^{-A\left(t \wedge \tau_{m}-s\right)} F u(s) d s \\
& +\int_{0}^{t \wedge \tau_{m}} e^{-A\left(t \wedge \tau_{m}-s\right)} G\left(y_{m}(s)\right) d L(s) .
\end{aligned}
$$

Since the sequence of stopping times $\left(\tau_{m}\right)_{m \in \mathbb{N}_{0}}$ is increasing and bounded, there exists a stopping time $\tau$ such that $\tau=\lim _{m \rightarrow \infty} \tau_{m}$. Moreover, we have $\mathbb{P}$-a.s. $0<\tau \leq T$. We set for each $m \in \mathbb{N}_{0}$, all $t \in\left[0, \tau_{m}\right)$ and $\mathbb{P}$-a.s.

$$
y(t)=y_{m}(t) .
$$

Then the process $(y(t))_{t \in[0, \tau)}$ is the unique local mild solution of system $[6$.

To finish this section, we give some remarks about the local mild solution of system 6 .

Remark 4.8 Note that the previous theorem is especially valid for $n=2$ and $n=3$. Hence, we get the existence and uniqueness of a solution to the stochastic Navier-Stokes equations for two-dimensional as well as three-dimensional domains up to a certain stopping time.

Remark 4.9 In contrast to the theory introduced in this section, one can also define a weak or a martingale solution to system [6, see [10, 11, 16]. For the case of a Wiener noise, we also refer to [8,9, 12, -15]. For these definitions, it is required that the solution takes values in the space $V$ introduced in Section 2 . It is well known that $V=D\left(A^{1 / 2}\right)$, see [2, 10, 28]. In this paper, the local mild solution of system 6 takes values in $D\left(A^{\alpha}\right)$ with $\alpha \in(0,1)$ and we have $D\left(A^{1 / 2}\right) \subset D\left(A^{\alpha}\right)$ for $\alpha \in\left(0, \frac{1}{2}\right)$ by Lemma 2.2. Thus, we constructed a solution well defined on a larger space, which is the main advantage of this approach. One may obtain that the local mild solution of system (6) is equivalent to a weak solution for the case $\alpha=\frac{1}{2}$.

Remark 4.10 (i) In case of additive noise in system (6), i.e. $G(y) \equiv G$, we have

$$
\mathbb{E} \sup _{t \in[0, \rho]}\|y(t)\|_{D\left(A^{\alpha}\right)}^{2}<\infty
$$

for a certain stopping time $\rho$ with values in $[0, T]$ and independent of $m \in \mathbb{N}_{0}$. The idea of the proof may be found in [19, 20].

(ii) If, in addition to the assumptions of Theorem 4.7 we require

$$
\mathbb{E} \sup _{t \in[0, \tau)} \int_{0}^{t}(t-s)^{-n / 4}|\nabla y(s)| d s<\infty
$$

and the Lévy process is given by a Q-Wiener process, then the solution of system (6) is a global mild solution in the sense that $\mathbb{P}(\tau=T)=1$, see [18].

\section{Existence and Uniqueness of Optimal Controls}

Throughout this section, let the parameters $\alpha \in(0,1), \delta \in[0,1)$ and $\beta \in[0, \alpha]$ be as in Theorem 4.6 and let the initial value $\xi \in L^{2}\left(\Omega ; D\left(A^{\alpha}\right)\right)$ be fixed. Moreover, let us denote the mild solution of system $(11)$ by $\left(y_{m}(t ; u)\right)_{t \in[0, T]}$ and the local mild solution of system $\left[6\right.$ by $(y(t ; u))_{t \in\left[0, \tau^{u}\right)}$ to illustrate the dependence on the control $u \in U$. Notice that the stopping times $\left(\tau_{m}^{u}\right)_{m \in \mathbb{N}_{0}}$ defined by 17 and the stopping time $\tau^{u}$ depend on the control $u \in U$ as well. Here, we consider the following cost functional for fixed $m \in \mathbb{N}_{0}$ and $\gamma \in[0, \alpha]$ :

$$
J_{m}(u)=\frac{1}{2} \mathbb{E} \int_{0}^{\tau_{m}^{u}}\left\|A^{\gamma}\left(y(t ; u)-y_{d}(t)\right)\right\|_{H}^{2} d t+\frac{1}{2} \mathbb{E} \int_{0}^{T}\left\|A^{\beta} u(t)\right\|_{H}^{2} d t,
$$


where $y_{d} \in L^{2}\left([0, T] ; D\left(A^{\gamma}\right)\right)$ is a given desired state and $u \in U$. The task is to find a control $\bar{u}_{m} \in U$ such that

$$
J_{m}\left(\bar{u}_{m}\right)=\inf _{u \in U} J_{m}(u)
$$

The control $\bar{u}_{m} \in U$ is called an optimal control. Note that for $\gamma=0$, the formulation coincides with the tracking problem, for more details see [23-26]. For $\gamma=\frac{1}{2}$ and $y_{d} \equiv 0$, we minimize the enstrophy, see [2 27, 28]. Hence, we are dealing with a generalized cost functional, which incorporates common control problems in fluid dynamics.

Remark 5.1 (i) Note that due to the definition of the local mild solution, we only can ensure that the first addend of the cost functional given by (19) is well defined up to the stopping time $\tau_{m}^{u}$ for fixed $m \in \mathbb{N}_{0}$.

(ii) In case of additive noise in system (6), we can replace the stopping $\tau_{m}^{u}$ in equation (19) by a certain stopping time $\rho^{u}$ independent of $m \in \mathbb{N}_{0}$.

(iii) If the assumptions of Remark 4.10 (ii) are fulfilled, then we can replace the stopping time $\tau_{m}^{u}$ in equation (19) by the deterministic terminal point of time $T$.

Next, we state the main result of this section.

Theorem 5.2 Let the functional $J_{m}(u)$ be given by (19) for fixed $m \in \mathbb{N}_{0}$ and arbitrary $u \in U$. Then there exists a unique optimal control $\bar{u}_{m} \in U$.

In the remaining part of this section, we give a proof of this result. Since the state as well as the stopping times are non-convex with respect to the control, we formulated a control problem using a non-convex cost functional. Therefore, we cannot prove the existence and uniqueness of the optimal control $\bar{u}_{m} \in U$ by well-known results for convex optimization problems. Here, we apply a result provided in [1.43]. For that purpose, we have to show that the cost functional is semi-continuous. In the following lemma, we prove that the state as well as the stopping times are continuous with respect to the control.

Lemma 5.3 For fixed $m \in \mathbb{N}_{0}$, let $\left(y_{m}(t ; u)\right)_{t \in[0, T]}$ be the mild solution of system $(11]$ corresponding to the control $u \in U$. Then there exists a constant $K>0$ such that for every $u_{1}, u_{2} \in U$ :

$$
\mathbb{E} \sup _{t \in[0, T]}\left\|y_{m}\left(t ; u_{1}\right)-y_{m}\left(t ; u_{2}\right)\right\|_{D\left(A^{\alpha}\right)}^{2} \leq K \mathbb{E} \int_{0}^{T}\left\|u_{1}(t)-u_{2}(t)\right\|_{D\left(A^{\beta}\right)}^{2} d t .
$$

Proof. Let $m \in \mathbb{N}_{0}$ be fixed and let $T_{1, m} \in(0, T]$. Similarly to inequalities $[14$, , $[15)$ and $[16)$, we obtain for every $u_{1}, u_{2} \in U$

$$
\begin{aligned}
& \mathbb{E} \sup _{t \in\left[0, T_{1, m}\right]}\left\|y_{m}\left(t ; u_{1}\right)-y_{m}\left(t ; u_{2}\right)\right\|_{D\left(A^{\alpha}\right)}^{2} \\
& \leq 3 \mathbb{E} \sup _{t \in\left[0, T_{1, m}\right]}\left\|\int_{0}^{t} e^{-A(t-s)} F\left[u_{1}(s)-u_{2}(s)\right] d s\right\|_{D\left(A^{\alpha}\right)}^{2} \\
& \quad+3 \mathbb{E} \sup _{t \in\left[0, T_{1, m}\right]}\left\|\int_{0}^{t} A^{\delta} e^{-A(t-s)} A^{-\delta}\left[B\left(\pi_{m}\left(y_{m}\left(s ; u_{1}\right)\right)\right)-B\left(\pi_{m}\left(y_{m}\left(s ; u_{2}\right)\right)\right)\right] d s\right\|_{D\left(A^{\alpha}\right)}^{2} \\
& \quad+3 \mathbb{E} \sup _{t \in\left[0, T_{1, m}\right]}\left\|\int_{0}^{t} e^{-A(t-s)}\left[G\left(y_{m}\left(s ; u_{1}\right)\right)-G\left(y_{m}\left(s ; u_{2}\right)\right)\right] d L(s)\right\|_{D\left(A^{\alpha}\right)}^{2} \\
& \leq 3 c_{2} \mathbb{E} \int_{0}^{T}\left\|u_{1}(t)-u_{2}(t)\right\|_{D\left(A^{\beta}\right)}^{2} d t+K_{1, m} \mathbb{E} \sup _{t \in\left[0, T_{1, m}\right]}\left\|y_{m}\left(t ; u_{1}\right)-y_{m}\left(t ; u_{2}\right)\right\|_{D\left(A^{\alpha}\right)}^{2},
\end{aligned}
$$


where $K_{1, m}=3 C_{1} m^{2} T_{1, m}^{2-2 \alpha-2 \delta}+3 C_{2} T_{1, m}$. We choose the point of time $T_{1, m} \in(0, T]$ such that $K_{1, m}<1$. Hence, we have for every $u_{1}, u_{2} \in U$

$$
\mathbb{E} \sup _{t \in\left[0, T_{1, m}\right]}\left\|y_{m}\left(t ; u_{1}\right)-y_{m}\left(t ; u_{2}\right)\right\|_{D\left(A^{\alpha}\right)}^{2} \leq c_{1, m} \mathbb{E} \int_{0}^{T}\left\|u_{1}(t)-u_{2}(t)\right\|_{D\left(A^{\beta}\right)}^{2} d t,
$$

where $c_{1, m}=\frac{3 c_{3}}{1-K_{1, m}}$. Next, we consider for all $t \in\left[T_{1, m}, T\right], \mathbb{P}$-a.s. and for $i=1,2$

$$
\begin{aligned}
y_{m}\left(t ; u_{i}\right)= & e^{-A\left(t-T_{1, m}\right)} y_{m}\left(T_{1, m} ; u_{i}\right)-\int_{T_{1, m}}^{t} A^{\delta} e^{-A(t-s)} A^{-\delta} B\left(\pi_{m}\left(y_{m}\left(s ; u_{i}\right)\right)\right) d s \\
& +\int_{T_{1, m}}^{t} e^{-A(t-s)} F u_{i}(s) d s+\int_{T_{1, m}}^{t} e^{-A(t-s)} G\left(y_{m}\left(s ; u_{i}\right)\right) d L(s) .
\end{aligned}
$$

Again, we find $T_{2, m} \in\left[T_{1, m}, T\right]$ such that for every $u_{1}, u_{2} \in U$

$$
\mathbb{E} \sup _{t \in\left[T_{1, m}, T_{2, m}\right]}\left\|y_{m}\left(t ; u_{1}\right)-y_{m}\left(t ; u_{2}\right)\right\|_{D\left(A^{\alpha}\right)}^{2} \leq c_{2, m} \mathbb{E} \int_{0}^{T}\left\|u_{1}(t)-u_{2}(t)\right\|_{D\left(A^{\beta}\right)}^{2} d t,
$$

where $c>0$ is a constant. Therefore, there exist points of time such that $0=T_{0, m}<T_{1, m}<\ldots<T_{l, m}=T$ and we have for $j=1, \ldots, l$

$$
\mathbb{E} \sup _{t \in\left[T_{j-1, m}, T_{j, m}\right]}\left\|y_{m}\left(t ; u_{1}\right)-y_{m}\left(t ; u_{2}\right)\right\|_{D\left(A^{\alpha}\right)}^{2} \leq c_{j, m} \mathbb{E} \int_{0}^{T}\left\|u_{1}(t)-u_{2}(t)\right\|_{D\left(A^{\beta}\right)}^{2} d t,
$$

where $c_{j, m}>0$ is a constant. Hence, we get

$$
\begin{aligned}
\mathbb{E} \sup _{t \in[0, T]}\left\|y_{m}\left(t ; u_{1}\right)-y_{m}\left(t ; u_{2}\right)\right\|_{D\left(A^{\alpha}\right)}^{2} & \leq \sum_{j=1}^{l} \mathbb{E} \sup _{t \in\left[T_{j-1, m}, T_{j, m}\right]}\left\|y_{m}\left(t ; u_{1}\right)-y_{m}\left(t ; u_{2}\right)\right\|_{D\left(A^{\alpha}\right)}^{2} \\
& \leq K \mathbb{E} \int_{0}^{T}\left\|u_{1}(t)-u_{2}(t)\right\|_{D\left(A^{\beta}\right)}^{2} d t
\end{aligned}
$$

where $K=\sum_{j=1}^{l} c_{j, m}$.

Lemma 5.4 For fixed $m \in \mathbb{N}_{0}$, let $\left(y_{m}(t ; u)\right)_{t \in[0, T]}$ be the mild solution of system $(11)$ corresponding to the control $u \in U$ and let the stopping time $\tau_{m}^{u}$ be defined by (17). Then we have

$$
\lim _{u_{1} \rightarrow u_{2}} \mathbb{P}\left(\tau_{m}^{u_{1}} \neq \tau_{m}^{u_{2}}\right)=0,
$$

where $u_{1} \rightarrow u_{2}$ is in the sense that $\mathbb{E} \int_{0}^{T}\left\|u_{1}(t)-u_{2}(t)\right\|_{D\left(A^{\beta}\right)}^{2} d t \rightarrow 0$.

Proof. By the extended version of Markov's inequality, and Lemma 5.3 , we get for all $\varepsilon>0$

$$
\begin{aligned}
\left.\mathbb{P}\left(\sup _{t \in[0, T]} \| y_{m}\left(t ; u_{1}\right)\right)-y_{m}\left(t ; u_{2}\right) \|_{D\left(A^{\alpha}\right)} \geq \varepsilon\right) & \left.\leq \frac{1}{\varepsilon^{2}} \mathbb{E} \sup _{t \in[0, T]} \| y_{m}\left(t ; u_{1}\right)\right)-y_{m}\left(t ; u_{2}\right) \|_{D\left(A^{\alpha}\right)}^{2} \\
& \leq \frac{K}{\varepsilon^{2}} \mathbb{E} \int_{0}^{T}\left\|u_{1}(t)-u_{2}(t)\right\|_{D\left(A^{\beta}\right)}^{2} d t
\end{aligned}
$$


Next, we assume $\lim _{u_{1} \rightarrow u_{2}} \mathbb{P}\left(\tau_{m}^{u_{1}}<\tau_{m}^{u_{2}}\right)>0$. Due to the definition of the stopping times, we can conclude

$$
\lim _{u_{1} \rightarrow u_{2}} \mathbb{P}\left(\left\{\left\|y_{m}\left(\tau_{m}^{u_{1}} ; u_{1}\right)\right\|_{D\left(A^{\alpha}\right)}>m\right\} \cap\left\{\left\|y_{m}\left(\tau_{m}^{u_{1}} ; u_{2}\right)\right\|_{D\left(A^{\alpha}\right)} \leq m\right\}\right)>0 .
$$

Therefore, there exists $\varepsilon_{0}>0$ such that

$$
\lim _{u_{1} \rightarrow u_{2}} \mathbb{P}\left(\left\|y_{m}\left(\tau_{m}^{u_{1}} ; u_{1}\right)\right\|_{D\left(A^{\alpha}\right)}-\left\|y_{m}\left(\tau_{m}^{u_{1}} ; u_{2}\right)\right\|_{D\left(A^{\alpha}\right)} \geq \varepsilon_{0}\right)>0
$$

This implies $\lim _{u_{1} \rightarrow u_{2}} \mathbb{P}\left(\left\|y_{m}\left(\tau_{m}^{u_{1}} ; u_{1}\right)-y_{m}\left(\tau_{m}^{u_{1}} ; u_{2}\right)\right\|_{D\left(A^{\alpha}\right)} \geq \varepsilon_{0}\right)>0$, which is a contradiction to 21. Hence, we get $\lim _{u_{1} \rightarrow u_{2}} \mathbb{P}\left(\tau_{m}^{u_{1}}<\tau_{m}^{u_{2}}\right)=0$. Similarly, we obtain $\lim _{u_{1} \rightarrow u_{2}} \mathbb{P}\left(\tau_{m}^{u_{1}}>\tau_{m}^{u_{2}}\right)=0$.

Lemma 5.5 Let $y_{d} \in L^{2}\left([0, T] ; D\left(A^{\gamma}\right)\right)$ be fixed. Moreover, let $(y(t ; u))_{t \in\left[0, \tau^{u}\right)}$ be the local mild solution of system (6) corresponding to the control $u \in U$, where the stopping times $\left(\tau_{m}^{u}\right)_{m \in \mathbb{N}_{0}}$ are defined by (17). Then for fixed $m \in \mathbb{N}_{0}$, the functional

$$
f_{m}(u)=\mathbb{E} \int_{0}^{\tau_{m}^{u}}\left\|A^{\gamma}\left(y(t ; u)-y_{d}(t)\right)\right\|_{H}^{2} d t
$$

is continuous with respect to the control $u \in U$.

Proof. Let the process $\left(y_{m}(t ; u)\right)_{t \in[0, T]}$ be the mild solution of system $[11)$ corresponding to the control $u \in U$ and let $u_{1}, u_{2} \in U$. We define the stopping times $\underline{\tau_{m}}=\tau_{m}^{u_{1}} \wedge \tau_{m}^{u_{2}}$ and $\frac{1}{\tau_{m}}=\tau_{m}^{u_{1}} \vee \tau_{m}^{u_{2}}$, and let the control $\bar{u} \in U$ be given by

$$
\bar{u}= \begin{cases}u_{1} & \text { if } \overline{\tau_{m}}=\tau_{m}^{u_{1}}, \\ u_{2} & \text { if } \overline{\tau_{m}}=\tau_{m}^{u_{2}} .\end{cases}
$$

Using Lemma 2.2, equation 18 , and the Cauchy-Schwarz inequality, there exists a constant $\widetilde{K}>0$ such that

$$
\begin{aligned}
\left|f_{m}\left(u_{1}\right)-f_{m}\left(u_{2}\right)\right|= & \left|\mathbb{E} \int_{0}^{\tau_{m}^{u_{1}}}\left\|A^{\gamma}\left(y_{m}\left(t ; u_{1}\right)-y_{d}(t)\right)\right\|_{H}^{2} d t-\mathbb{E} \int_{0}^{\tau_{m}^{u_{2}}}\left\|A^{\gamma}\left(y_{m}\left(t ; u_{2}\right)-y_{d}(t)\right)\right\|_{H}^{2} d t\right| \\
\leq & \mathbb{E} \int_{0}^{\frac{\tau_{m}}{\tau_{m}} \mid}\left\|A^{\gamma}\left(y_{m}\left(t ; u_{1}\right)-y_{d}(t)\right)\right\|_{H}^{2}-\left\|A^{\gamma}\left(y_{m}\left(t ; u_{2}\right)-y_{d}(t)\right)\right\|_{H}^{2} \mid d t \\
& +\mathbb{E} \int_{\overline{\tau_{m}}}\left\|A^{\gamma}\left(y_{m}(t ; \bar{u})-y_{d}(t)\right)\right\|_{H}^{2} d t \\
\leq & \widetilde{K}\left(\mathbb{E} \sup _{t \in[0, T]}\left\|y_{m}\left(t ; u_{1}\right)-y_{m}\left(t ; u_{2}\right)\right\|_{D\left(A^{\alpha}\right)}^{2}\right)^{1 / 2} \\
& \left.+2 \int_{0}^{T} \mathbb{P}\left(\tau_{m}^{u_{1}} \wedge \tau_{m}^{u_{2}} \leq t<\tau_{m}^{u_{1}} \vee \tau_{m}^{u_{2}}\right)\left(C^{2} m^{2}+\| y_{d}(t)\right) \|_{D\left(A^{\gamma}\right)}^{2}\right) d t .
\end{aligned}
$$

Due to Lemma 5.3 , we have $\lim _{u_{1} \rightarrow u_{2}} \mathbb{E} \sup _{t \in[0, T]}\left\|y_{m}\left(t ; u_{1}\right)-y_{m}\left(t ; u_{2}\right)\right\|_{D\left(A^{\alpha}\right)}^{2}=0$. By Lemma 5.4, we get $\lim _{u_{1} \rightarrow u_{2}} \mathbb{P}\left(\tau_{m}^{u} \wedge \tau_{m}^{u_{2}} \leq t<\tau_{m}^{u_{1}} \vee \tau_{m}^{u_{2}}\right)=0$. Using Lebesgue's dominated convergence theorem, we obtain

$$
\lim _{u_{1} \rightarrow u_{2}}\left|f_{m}\left(u_{1}\right)-f_{m}\left(u_{2}\right)\right|=0 .
$$

Hence, the functional $f_{m}(u)$ is continuous with respect to the control $u \in U$. 
To find a unique optimal control $\bar{u}_{m} \in U$ satisfying [20], we use the following result.

Proposition 5.6 (cf. [143]) Let $\mathcal{B}$ be a uniformly convex Banach space and let $V \subset \mathcal{B}$ be bounded and closed. Moreover, let $f: V \rightarrow \mathbb{R} \cup\{-\infty, \infty\}$ be a lower semi-continuous functional, which is bounded from below. Then there exists a dense subset $V_{0} \subset V$ such that for any $v \in V_{0}$ and any $q \geq 1$ the functional $f(u)+\|u-v\|_{\mathcal{B}}^{q}$ attains its minimum over $V$, i.e. there exists $u(v) \in V$ such that

$$
f(u(v))+\|u(v)-v\|_{\mathcal{B}}^{q}=\inf _{u \in V}\left(f(u)+\|u-v\|_{\mathcal{B}}^{q}\right) .
$$

If $q>1$, then $u(v)$ is unique. Furthermore, the function $v \mapsto u(v)$ is continuous.

We are now able to prove the existence and uniqueness of the optimal controls.

Proof of Theorem 5.2. The space $L^{2}\left(\Omega ; L^{2}\left([0, T] ; D\left(A^{\beta}\right)\right)\right)$ is a Hilbert space and thus, a uniformly convex Banach space. Moreover, the set of admissible controls $U$ is a subset of the space $L^{2}\left(\Omega ; L^{2}\left([0, T] ; D\left(A^{\beta}\right)\right)\right)$ and by definition bounded and closed. Due to Lemma 5.5, the functional

$$
f_{m}(u)=\mathbb{E} \int_{0}^{\tau_{m}^{u}}\left\|A^{\gamma}\left(y(t ; u)-y_{d}(t)\right)\right\|_{H}^{2} d t
$$

is lower semi-continuous and we have $f_{m}(u) \geq 0$ for every $u \in U$. Applying Proposition 5.6, we get the existence of a dense subset $V_{0} \subset U$ such that for any $v \in V_{0}$ the functional $\frac{1}{2} f_{m}(u)+\frac{1}{2}\|u-v\|_{L^{2}\left(\Omega ; L^{2}\left([0, T] ; D\left(A^{\beta}\right)\right)\right.}^{2}$ attains its minimum over $U$. We denote the minimum by $u_{m}(v) \in U$ and due to Proposition 5.6, it is unique and continuous with respect to $v \in V_{0}$. Since $V_{0}$ is a dense subset of $U$, there exists a sequence $\left(v_{k}\right)_{k \in \mathbb{N}} \subset V_{0}$ such that $\lim _{k \rightarrow \infty}\left\|v_{k}\right\|_{L^{2}\left(\Omega ; L^{2}\left([0, T] ; D\left(A^{\beta}\right)\right)\right)}=0$. We define $\bar{u}_{m}=\lim _{k \rightarrow \infty} u_{m}\left(v_{k}\right)$. Due to the continuity properties, we get

$$
\begin{aligned}
J_{m}\left(\bar{u}_{m}\right) & =\lim _{k \rightarrow \infty}\left(\frac{1}{2} f_{m}\left(u_{m}\left(v_{k}\right)\right)+\frac{1}{2}\left\|u_{m}\left(v_{k}\right)-v_{k}\right\|_{L^{2}\left(\Omega ; L^{2}\left([0, T] ; D\left(A^{\beta}\right)\right)\right)}^{2}\right) \\
& =\lim _{k \rightarrow \infty} \inf _{u \in U}\left(\frac{1}{2} f_{m}(u)+\frac{1}{2}\left\|u-v_{k}\right\|_{L^{2}\left(\Omega ; L^{2}\left([0, T] ; D\left(A^{\beta}\right)\right)\right)}^{2}\right) \\
& =\inf _{u \in U}\left(\frac{1}{2} f_{m}(u)+\frac{1}{2}\|u\|_{L^{2}\left(\Omega ; L^{2}\left([0, T] ; D\left(A^{\beta}\right)\right)\right)}^{2}\right) \\
& =\inf _{u \in U} J_{m}(u) .
\end{aligned}
$$

Therefore, we get the existence and uniqueness of the optimal control $\bar{u}_{m} \in U$ satisfying (20).

Remark 5.7 Here, we analyze the cost functional $J_{m}(\cdot)$ given by $(19)$ for $m \in \mathbb{N}_{0}$. Therefore, the optimal controls $\bar{u}_{m} \in U$ also depend on $m \in \mathbb{N}_{0}$ and we get a sequence of optimal controls. Note that the initial aim is to control the stochastic Navier-Stokes equations in a desired way. Thus, it is reasonable to design an optimal control $\bar{u} \in U$ independent of $m \in \mathbb{N}_{0}$ but still linked to the optimal control $\bar{u}_{m} \in U$ for each $m \in \mathbb{N}_{0}$, for instance $\bar{u}(t)=\bar{u}_{m}(t)$ for almost all $t \in\left[0, \tau_{m}\right)$ in some sense.

\section{Conclusions}

In this paper, we have considered the controlled multidimensional stochastic Navier-Stokes equations on bounded domains with multiplicative Lévy noise. Under certain conditions on the parameters, we proved the existence and uniqueness of the state in the sense of a local mild solution. A global mild solution can be obtained by additional assumptions. The cost functional introduced in this paper is motivated by common control problems of the Navier-Stokes equations. We proved continuity results for the state as well as for the stopping times with respect to the control to infer the continuity of the cost functional. Finally, we obtained the existence and uniqueness of optimal controls based on [1].

In future work, we will derive necessary and sufficient optimality conditions optimal controls have to satisfy. Therefore, we will derive explicit formulas for the optimal controls. 


\section{Acknowledgement}

This research is supported by a research grant of the 'International Max Planck Research School (IMPRS) for Advanced Methods in Process and System Engineering', Magdeburg. The authors would like to thank Prof. Wilfried Grecksch of the Martin Luther University Halle-Wittenberg for his helpful advice on various technical issues.

\section{References}

[1] M.F. Bidaut, Théorème d'existence et d'existence "en général" d'un contrôle optimal pour des systèmes régis par des équations aux dérivées partielles non linéaires, PhD thesis, Université Paris VI, 1973.

[2] S. S. Sritharan, in: Optimal Control of Viscous Flow (SIAM, Philadelphia, 1998), chap. 1. An Introduction to Deterministic and Stochastic Control of Viscous Flow, pp. 1-42.

[3] V. Barbu, Stabilization of Navier-Stokes Flow (Springer, London, 2011).

[4] E. Hopf, Über die Anfangswertaufgabe für die hydrodynamischen Grundgleichungen, Math. Nachr. 4, 213-231 (1950).

[5] H. Fujita and T. Kato, On the Navier-Stokes initial value problem. I, T. Arch. Rational Mech. Anal. 16(4), 269-315 (1964).

[6] Y. Giga, Weak and Strong Solutions of the Navier-Stokes Initial Value Problem, Publ. Res. Inst. Math. Sci. 19, 887910 (1983).

[7] Y. Giga and T. Miyakawa, Solutions in $L_{r}$ of the Navier-Stokes initial value problem, Arch. Ration. Mech. Anal. 89(3), 267-281 (1985)

[8] J. L. Menaldi and S. S. Sritharan, Stochastic 2-D Navier-Stokes Equation, Appl. Math. Optim. 46, 31-53 (2002).

[9] S. S. Sritharan and P. Sundar, Large deviations for the two-dimensional Navier-Stokes equations with multiplicative noise, Stochastic Process. Appl. 116, 1636-1659 (2006).

[10] Z. Brzeźniak, E. Hausenblas, and J. Zhu, 2D stochastic Navier-Stokes equations driven by jump noise, Nonlinear Anal. 79, 122-139 (2013).

[11] B.P. W. Fernando and S. Sritharan, Nonlinear filtering of stochastic Navier-Stokes equation with Itô-Lévy noise, Stoch. Anal. Appl. 31(3), 381-426 (2013).

[12] H. Breckner, Galerkin approximation and the strong solution of the Navier-Stokes equation, J. Appl. Math. Stoch. Anal. 13(3), 239-259 (2000).

[13] M. Capinski and D. Gaterek, Stochastic equations in Hilbert space with application to Navier-Stokes equations in any dimension, J. Funct. Anal. 126, 26-35 (1994).

[14] F. Flandoli, in: Proceedings of the CIME Course on SPDE in Hydrodynamics: Recent Progress and Prospects. Lecture Notes in Mathematics (Springer, Berlin, 2008), chap. 2. An Introduction to 3D Stochastic Fluid Dynamics, pp. 51-150.

[15] F. Flandoli and D. Gatarek, Martingale and stationary solutions for stochastic Navier-Stokes equations, Probab. Theory Relat. Fields 102, 367-391 (1995).

[16] E. Motyl, Stochastic Navier-Stokes equations driven by Lévy noise in unbounded 3D domains, Potential Anal. 38, 863-912 (2013).

[17] G. Da Prato and J. Zabczyk, Ergodicity for Infinite Dimensional Systems (Cambridge University Press, 1996).

[18] L. Debbi, Well-Posedness of the Multidimensional Fractional Stochastic Navier-Stokes Equations on the Torus and on Bounded Domains, J. Math. Fluid Mech. 18, 25-69 (2016).

[19] A. Bensoussan and J. Frehse, Local Solutions for Stochastic Navier Stokes Equations, M2AN Math. Model. Numer. Anal. 34(2), 241-273 (2000).

[20] B.P.W. Fernando, B. Rüdiger, and S. S. Sritharan, Mild solutions of stochastic Navier-Stokes equation with jump noise in $\mathbb{L}^{p}$-spaces, Math. Nachr. 288, 1615-1621 (2015).

[21] M. T. Mohan and S. S. Sritharan, $\mathbb{L}^{p}$-solutions of the stochastic Navier-Stokes equations subject to Lévy noise with $\mathbb{L}^{m}\left(\mathbb{R}^{m}\right)$ initial data, Evol. Equ. Control Theory 3, 409-425 (2017).

[22] N. Glatt-Holtz and M. Ziane, Strong pathwise solutions of the stochastic Navier-Stokes system, Adv. Differential Equations 14(5-6), 567-600 (2009).

[23] M. Hintermüller and M. Hinze, A SQP-Semismooth Newton-type Algorithm applied to Control of the instationary Navier-Stokes System Subject to Control Constraints, SIAM J. Optim. 16(4), 1177-1200 (2006).

[24] H. Liang, , L. Hou, and J. Ming, The velocity tracking problem for Wick-stochastic Navier-Stokes flows using Wiener chaos expansion, J. Comput. Appl. Math. 307, 25-36 (2016).

[25] Y. R. Ou, in: Optimal Control of Viscous Flow (SIAM, Philadelphia, 1998), chap. 7. Design of Feedback Compensators for Viscous Flow, pp. 151-180.

[26] M. Ulbrich, Constrained optimal control of Navier-Stokes flow by semismooth Newton methods, Systems Control Lett. 48, 297-311 (2003). 
[27] G. Da Prato and A. Debussche, Dynamic Programming for the stochastic Navier-Stokes equations, M2AN Math Model. Numer. Anal. 34(2), 459-475 (2000).

[28] F. Gozzi, S. S. Sritharan, and A. Święch, Bellman Equations Associated to the Optimal Feedback Control of Stochastic Navier-Stokes Equations, Comm. Pure Appl. Math. 58(5), 671-700 (2005).

[29] D. Wachsmuth, Regularity and stability of optimal controls of nonstationary Navier-Stokes equations, Control and Cybernetics 34(2), 387-409 (2005).

[30] D. Fujiwara and H. Morimoto, An $L_{r}$-theorem of the Helmholtz decomposition of vector fields, J. Fac. Sci. Univ. Tokyo Sec. 1 A 24(3), 685-700 (1977).

[31] H. Fujita and H. Morimoto, On fractional powers of the Stokes operator, Proc. Japan Acad. 46, 1141-1143 (1970).

[32] Y. Giga, Analyticity of the Semigroup Generated by the Stokes Operator in $L_{r}$ Space, Math. Z. 178, 297-329 (1981).

[33] I. Vrabie, $C_{0}-$ Semigroups and Applications (Mathematics Studies no. 191. Elsevier, Amsterdam, 2003).

[34] A. Pazy, Semigroups of Linear Operators and Applications to Partial Differential Equations (Springer, New York, 1983).

[35] W. von Wahl, The Equations of Navier-Stokes and Abstract Parabolic Equations (Vieweg + Teubner Verlag, 1985).

[36] D. Fujiwara, Concrete characterization of the domains of fractional powers of some elliptic differential operators of the second order, Proc. Japan Acad. 43(2), 82-86 (1967).

[37] S. Peszat and J. Zabczyk, Stochastic Partial Differential Equations with Lévy Noise. An evolution equation approach (Encyclopedia of Mathematics and Its Applications 113. Cambridge University Press, Cambridge, 2007).

[38] D. Applebaum, Lévy Processes and Stochastic Calculus (Cambridge Studies in Advanced Mathematics 116. Cambridge: Cambridge University Press, 2009)

[39] G. Da Prato and J. Zabczyk, Stochastic Equations in Infinite Dimensions (Cambridge University Press, 2014).

[40] E. Hausenblas and J. Seidler, Stochastic Convolutions Driven by Martingales: Maximal Inequalities and Exponential Integrability, Stoch. Anal. Appl. 26, 98-119 (2007).

[41] Z. Brzeźniak, B. Maslowski, and J. Seidler, Stochastic nonlinear beam equations, Probab. Theory Relat. Fields 132, 119-149 (2005).

[42] Z. Brzeźniak and A. Carroll, in: Séminaire de Probabilités XXXVII, Lecture Notes in Mathematics, vol. 1832 (Springer-Verlag, Berlin, 2003), chap. 11. Approximations of the Wong-Zakai type for stochastic differential equations in M-type 2 Banach spaces with applications to loop spaces, pp. 251-289.

[43] M. Goebel, On Existence of Optimal Control, Math. Nachr. 93, 67-73 (1979). 